BULL. AUSTRAL. MATH. SOC.

$52 A 40,52 A 10$

VOL. 19 (1978), 131-133.

\title{
Two inequalities for convex sets in the plane
}

\section{P.R. Scott}

Let $K$ be a bounded, closed, convex set in the euclidean plane having diameter $d$, width $w$, inradius $r$, and circumradius $R$. We show that

$$
(w-2 r) d \leq 2 \sqrt{3} r^{2}
$$

and

$$
w(2 R-d) \leq \sqrt{3}(2-\sqrt{3}) R^{2}
$$

where both these inequalities are best possible.

Let $K$ be a bounded, closed, convex set in the euclidean plane. We denote the area, perimeter, diameter, (minimal) width, inradius, and circumradius of $K$ by $A, p, d, w, r$, and $R$ respectively. There are many known inequalities amongst the quantities $A, p, d, v, r$, and $R$ (see, for example, [1], [2]). The two inequalities established in the present paper appear to be new.

THEOREM 1. $(w-2 r) d \leq 2 \sqrt{3} r^{2}$, with equality when and only winen $K$ is an equilateral triangle of side length $2 \sqrt{3} \mathrm{r}$.

Proof. We observe that a largest circle inscribed in $K$ must either contain two boundary points of $K$ which are ends of a diameter of the circle, or else it contains three boundary points $U, V, W$ of $K$ which form the vertices of an acute angled triangle (see, for example, [3]).

In the first case, $w=2 r$, and the theorem is trivially true. In

Received 24 July 1978. 
the second case, the tangents to the circle at $U, V, V$ form a triangle, $\triangle X Y Z$ say. Since $K$ is convex, $K$ is contained in $\triangle X Y Z$. In fact, since we are interested in maximizing the width and diameter of $K$, we may take $K$ to be $\triangle X Y Z$.

We notice that for a triangle, the diameter is the length of a longest side, and the width is the altitude to that side. Thus

$$
\omega d=2 A=p r
$$

and

$$
(w-2 r) d=r(p-2 d) \text {. }
$$

Hence it is sufficient for us to maximize $p-2 d$ for a fixed value of $r$.

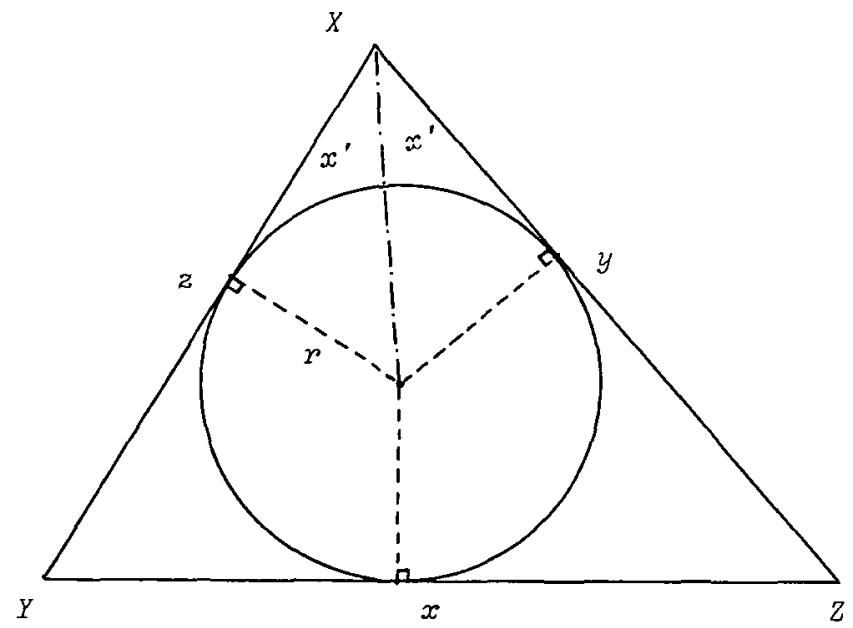

Using the notation in the diagram, let us assume that $x \geq y \geq z$. Now

$$
\begin{aligned}
p-2 d & =(x+y+z)-2 x \\
& =y+z-x \\
& =2 x^{\prime} .
\end{aligned}
$$

Since $r=x^{\prime} \tan (X / 2)$, and $r$ is fixed, the maximum value of $x^{\prime}$ will be assumed when $\mathscr{L}$ is as small as possible, subject to the constraint $x \geq y \geq z$. This occurs when $\underline{I X}=\pi / 3 \quad(=\underline{Y}=\mid \underline{Z})$; that is, when and only when $\triangle Y Y Z$ is equilateral. For this equilateral triangle, 
$d=2 \sqrt{3} r, \quad w=3 r, \quad(w-2 r) d=2 \sqrt{3} r^{2}$.

Hence for any convex set $i$,

$$
(w-2 r) d \leq 2 \sqrt{3} r^{2},
$$

as required.

THEOREM 2. $w(2 R-d) \leq \sqrt{3}(2-\sqrt{3}) R^{2}$, with equality when and only when $K$ is a Reuleaux triangle of width $\sqrt{3} R$.

Proof. It is known [3] that if $K$ has circumradius $R$, then $\sqrt{3} R \leq d \leq 2 R$. Also, $w \leq d$, so for any $d$,

$$
(2 R-d) \omega \leq(2 R-d) d \text {. }
$$

Now $f(d)=(2 R-d) d$ is a decreasing function of $d$, and so takes its maximum value for $d=\sqrt{3} R$. Hence

$$
(2 R-d) w \leq(2-\sqrt{3}) \sqrt{3} R^{2} .
$$

For equality here we require a set $K$ having $w=d$; that is, $K$ must be a set of constant width. Finally, it is known, [3], that the only set of constant width which satisfies $d=\sqrt{3} R$ is the Reuleaux triangle of width $\sqrt{3} R$. This completes the proof.

\section{References}

[1] 0. Bottema, R.ž. Djordjević, R.R. Janić, D.S. Mitrinović, P.M. Vasić, Geometric inequalities (Wolters-Noordhoff, Groningen, 1969).

[2] Marlow Sholander, "On certain minimum problems in the theory of convex curves", Trons. Amer. Math. Soc. 73 (1952), 139-173.

[3] I.M. Yaglom and V.G. BoltyanskiT, Convex figures (translated by Paul $J$. Kelly and Lewis F. Walton. Holt, Rinehart and Winston, New York, 1961).

Department of Pure Mathematics,

University of Adelaide,

Adelaide,

South Australia. 